\title{
NEONATO CON VENTRICULITIS CAUSADA POR Empedobacter brevis: REPORTE DE UN CASO EN PERÚ
}

\author{
Christian Rojas-Contreras ${ }^{1, a}$, Gabriel De la Cruz-Ku 2,b, Miguel Eduardo Eyzaguirre-Sandoval 2,b, \\ Bryan Valcarcel-Valdivia ${ }^{2, b}$
}

\begin{abstract}
RESUMEN
Las infecciones causadas por microorganismos poco comunes son objeto de investigación, ya que animar a los investigadores a encontrar las medidas sanitarias necesarias para prevenir y tratar la enfermedad, así como la búsqueda de nuevas luces sobre las interacciones humano-microbios. En este informe se describe el caso de un recién nacido varón diagnosticado de hidrocefalia y mielomeningocele, que desarrolló ventriculitis y sepsis por Empedobacter brevis resistente. Este caso pone de manifiesto la inesperada identificación de esta bacteria en el líquido cefalorraquídeo y su patrón multirresistente, que fue crucial para dar un manejo terapéutico adecuado. Esta bacteria evidencia una mezcla de diferentes etiologías en el análisis del líquido cefalorraquídeo.
\end{abstract}

Palabras clave: Infección; Ventriculitis cerebral; Recién nacido; Carbapenémicos; Colistina (fuente: DeCS BIREME).

\section{NEONATE WITH VENTRICULITIS CAUSED BY Empedobacter brevis: REPORT OF A CASE IN PERU}

\begin{abstract}
Infections caused by rare micro-organisms are the subject of research, as researchers are encouraged to find the necessary health measures to prevent and treat the disease, as well as the search for new insights into human-microbial interactions. This report describes the case of a newborn boy diagnosed with hydrocephalus and myelomeningocele who developed ventriculitis and sepsis from resistant Empedobacter brevis. This case highlights the unexpected identification of this bacterium in the cerebrospinal fluid and its multi-resistant pattern, which was crucial for proper therapeutic management. This bacterium shows a mixture of different etiologies in the analysis of cerebrospinal fluid.
\end{abstract}

Keywords: Infection; Cerebral ventriculitis; Infant, newborn; Carbapenems; Colistin (source: MeSH NLM).

\section{INTRODUCCIÓN}

Empedobacter brevis, previamente denominado Flavobacterium breve, es un bacilo Gram-negativo incluido en la familia de las Flavobacteriaceae ${ }^{(1)}$. Este microrganismo poco frecuente se encuentra en las secreciones oculares, las secreciones bronquiales, los hemocultivos y la orina, con una leve patogenicidad ${ }^{2,3)}$. Sin embargo, es resistente a las cefalosporinas y carbapenemas de espectro extendido, debido a la producción de ß-lactamasas ${ }^{(3,4)}$. Además, estudios previos refirieren una resistencia intermedia a amoxicilina y ticarcilina ${ }^{(2,5)}$. Este microorganismo es extremadamente raro en la población neonatal ( ${ }^{(6)}$. Ante ello, se presenta un neonato con mielomeningocele que desarrolló ventriculitis y sepsis por Empedobacter brevis.

\section{REPORTE DE CASO}

Se admitió en el Instituto Nacional de Salud del Niño un recién nacido de seis días de edad, procedente de Sullana, PiuraPerú, con un diagnóstico de sepsis por mielomeningocele infectado. Nació por cesárea de una madre de 38 años, tuvo diagnóstico intrauterino de mielomeningocele e hidrocefalia. El peso al nacer fue de $3,130 \mathrm{~kg}$, con 39 semanas de edad gestacional, $50 \mathrm{~cm}$ longitud, $33 \mathrm{~cm}$ perímetro cefálico y la puntuación de Apgar fue de 9/10 en el 1er y 5to minuto. Posteriormente, presentó dificultad respiratoria y respuesta letárgica al estímulo doloroso. El examen físico mostró fontanelas tensas y abombadas. Fue hospitalizado desde su primer día de vida por un mielomeningocele infectado, donde se administró ampicilina y amikacina. En su cuarto

\footnotetext{
Servicio de Infectología. Instituto Nacional de Salud del Niño. Lima, Perú.

Sociedad científica de estudiantes de Medicina Humana de la Universidad Científica del Sur, Universidad Científica del Sur. Lima, Perú.

Especialista en Enfermedades Infecciosas. ${ }^{\mathrm{b}}$ Estudiante de Medicina Humana.

Recibido: 03/09/2017 Aprobado: 21/03/2018 En línea: 09/07/2018
} 
día de vida, una ecocardiografía cerebral mostró hidrocefalia asimétrica severa.

Después de dos días fue referido al Instituto Nacional de Salud del Niño para realizar una reparación de mielomeningocele. A la admisión el paciente se encontró irritable, su peso fue de tres $\mathrm{kg}$, la temperatura axilar $38^{\circ} \mathrm{C}$, la frecuencia cardíaca 157 latidos por minuto, la frecuencia respiratoria 62 respiraciones por minuto, y la saturación de oxígeno del $99 \%$ respirando aire ambiental. El examen físico evidenció la fontanela anterior abombada e hipotonía generalizada, por lo que se diagnosticó ventriculitis. La cirugía se realizó con éxito en un período de dos horas y 20 minutos, después de eso se transfirió a la Unidad de Cuidados Intensivos para monitoreo constante.

Al día siguiente, $7 \mathrm{mo}$ día de vida, el paciente continuó irritable y febril. Se solicitó una resonancia magnética, la cual diagnosticó una malformación Arnold-Chiari tipo II y una heterotopía cortical subependimal (Figura 1). Además,

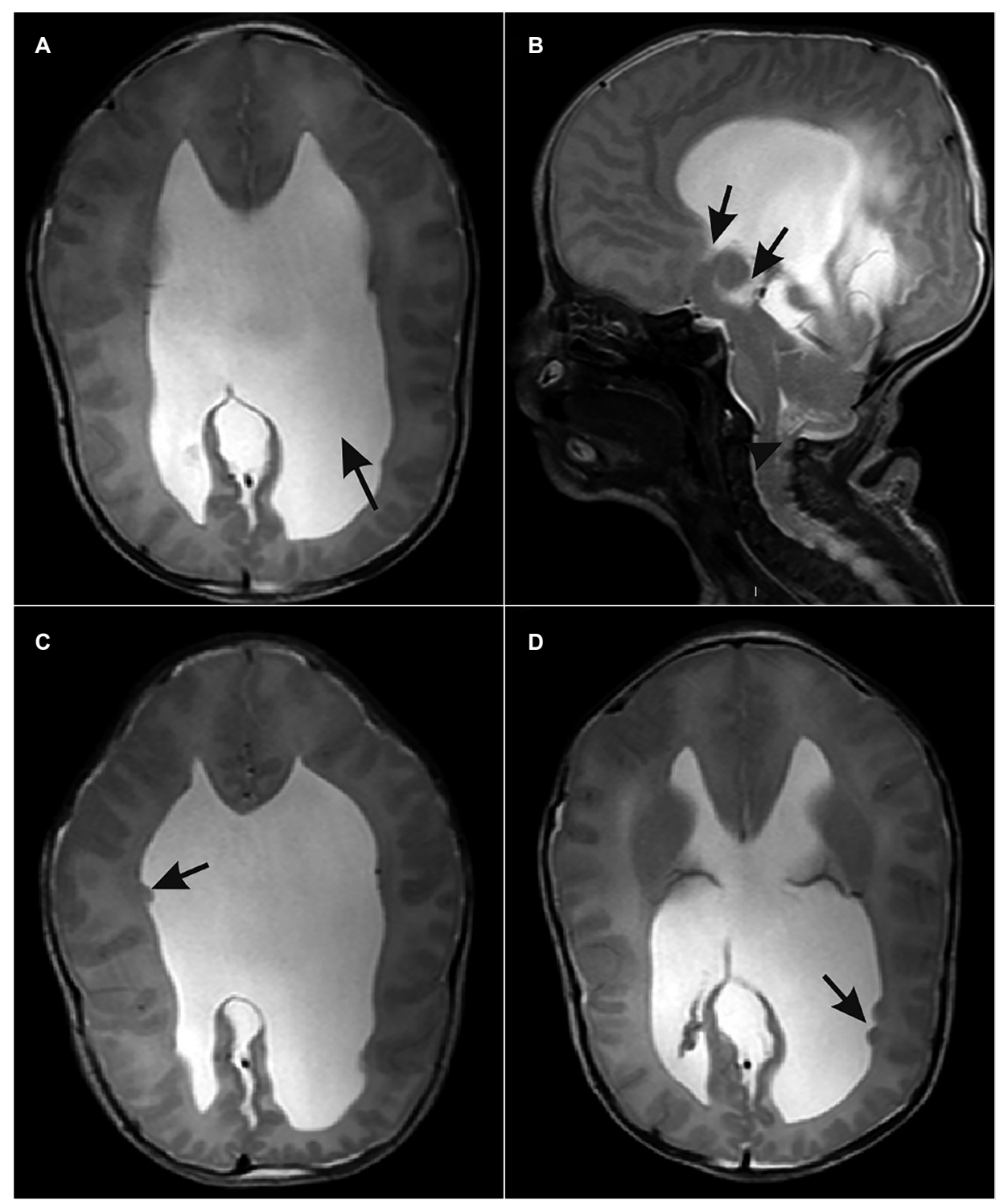

Figura 1. Recién nacido de $\mathrm{x}$ días, con mielomeningocele operado a los dos días de vida complicado con ventriculitis por Empedobacter brevis. La imagen de resonancia magnética (RM) del cerebro en el ingreso hospitalario muestra dilatación del sistema ventricular supratentorial fluido-ponderado T2 con dominancia del ventrículo izquierdo (panel A, flecha). También mostró una alteración de la señalización-disgénesis ponderada en T2 del cuerpo calloso, caracterizada por adelgazamiento progresivo antero-lateral (panel B, flechas) y señalización anormal marcada con T2- hernia inferior del bulbo y amígdalas situadas $13 \mathrm{~mm}$ por debajo del foramen magnum (Panel B, punta de flecha). En los paneles C y D (flechas) se observa una señalización anómala en T2 con pequeñas imágenes subependimales nodulares bilaterales, cuatro localizadas en el cuerno posterior lateral izquierdo del ventrículo, dos en el cuerno posterior y una en el derecho cuerpo ventricular lateral. 
se tomaron cultivos de sangre y líquido cefalorraquídeo (LCR). El LCR fue xantocrómico, nublado, recuento sanguíneo $289 \mathrm{cel} / \mathrm{mm}^{3}$, la glucosa $20 \mathrm{mg} / \mathrm{dL}$, las proteínas totales $110,23 \mathrm{mg} / \mathrm{dL}$, el test de Pandy positivo y se aisló bacilos gram-negativos. Se inició tratamiento empírico con meropenem y vancomicina. Al 8vo día de vida, se realizó un drenaje ventricular externo (DVE), para aliviar la hipertensión intracraneal. Después de cuatro días, el hemocultivo y cultivo de LCR identificaron al patógeno como Empedobacter brevis, con alta resistencia a los antibióticos (Tabla 1). No se efectuó una confirmación mediante el uso de PCR. Se inició tratamiento con piperacilina/tazobactam y amikacina, mientras se suspendió vancomicina y meropenem. De pronto, el paciente presentó convulsión tónico-clónica en 3 oportunidades no asociado con fiebre, se indicó fenobarbital.

Después de diez días de tratamiento antibiótico, el cultivo de sangre y LCR resultaron negativos. A pesar del abordaje terapéutico, no se evidenció mejora clínica, en pruebas laboratoriales y de LCR; por lo tanto, se rotó de antibiótico a meropenem más colistina. Las convulsiones persistieron, por lo que se agregó midazolam más fenitoína, con adecuada respuesta. A los 80 días de vida, se realizó una neuroendoscopia más fenestración intraventicular del septo y otra sustitución de la DVE. Los hallazgos mostraron predominio ventricular izquierdo de los tabiques, aislamiento del cuerno frontal y temporal, y varias petequias en la superficie ependimaria. Después de la cirugía, el paciente continuó su tratamiento habitual hasta la resolución de los síntomas. Los fármacos fenobarbital y fenitoína siempre fueron monitoreados y nunca alcanzaron los niveles tóxicos. Adicionalmente, la prueba de adenosina desaminasa (ADA) fue negativa en todos los análisis de LCR realizados. Esta prueba se efectuó como indicador de infección por Mycobacterium tuberculosis (Tabla 2).

El paciente pasó por ocho reemplazos de la DVE, una EVD y una neuroendoscopia más intraventricular septum fenestración; un total de diez procedimientos quirúrgicos. Fue dado de alta a los 90 días de vida, después de la remisión bacteriológica, bioquímica y sintomatológica de la enfermedad, sin presentar secuelas motoras o sensoriales.

Tabla 1. Susceptibilidad a los antimicrobianos de Empedobacter brevis aislado de sangre y líquido cefalorraquídeo de un recién nacido con ventriculitis

\begin{tabular}{|c|c|c|}
\hline Antibiótico & Concentración inhibitoria mínima (mg/L) & Interpretación \\
\hline Amikacina & $\leq 16$ & $S$ \\
\hline Amoxicillina/ácido clavulánico & $16 / 8$ & \\
\hline Ampicillina/sulbactam & $>16 / 8$ & \\
\hline Ampicillina & $>16$ & \\
\hline Cefazolina & $>4$ & \\
\hline Cefepime & $>16$ & $\mathrm{R}$ \\
\hline Cefotaxima & $>32$ & $\mathrm{R}$ \\
\hline Cefotaxima/ácido clavulánico & 4 & \\
\hline Ceftazidima & $>16$ & $\mathrm{R}$ \\
\hline Ceftazidima/ácido clavulánico & $>2$ & \\
\hline Ceftriaxona & $>32$ & $\mathrm{R}$ \\
\hline Cefuroxima & $>16$ & \\
\hline Ciprofloxacino & $\leq 1$ & S \\
\hline Ertapenem & $>2$ & \\
\hline Gentamicina & 4 & S \\
\hline Imipenem & $>8$ & $\mathrm{R}$ \\
\hline Levofloxacino & $\leq 2$ & S \\
\hline Meropenem & $>8$ & $\mathrm{R}$ \\
\hline Piperacillina/tazobactam & $\leq 16$ & S \\
\hline Tetraciclina & 8 & I \\
\hline Tobramicine & $>8$ & $\mathrm{R}$ \\
\hline Sulfametoxazole/trimetropin & $\leq 2 / 38$ & S \\
\hline
\end{tabular}

S: sensible, R: resistente, I: intermedio 


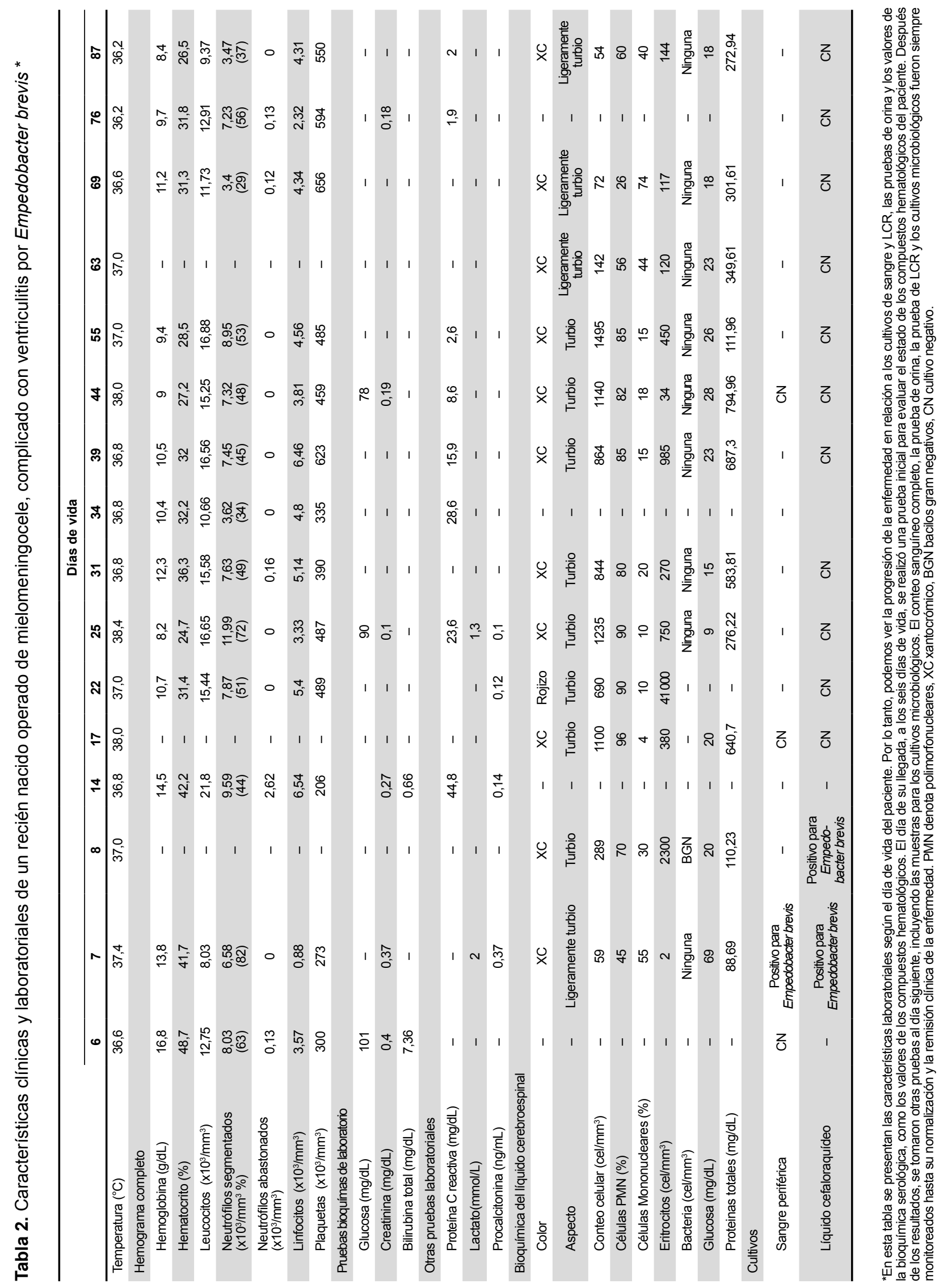




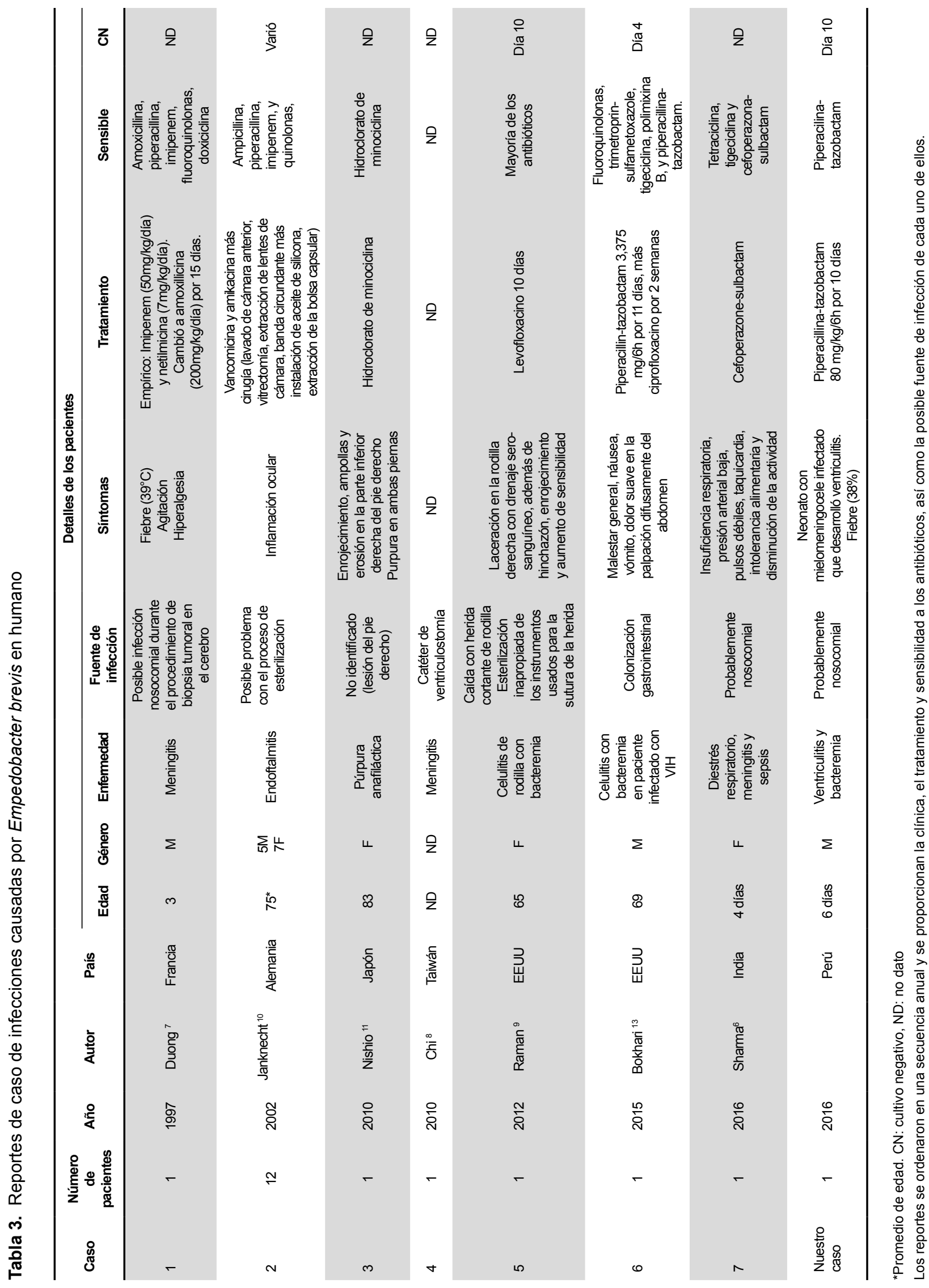




\section{DISCUSIÓN}

Se presenta el caso de un recién nacido con diagnóstico prenatal de hidrocefalia y mielomeningocele, que desarrolló ventriculitis y sepsis, causada por Empedobacter brevis. La información sobre la infección causada por esta bacteria es escasa, con sólo ocho artículos previos, siete en humanos y uno en un perro ${ }^{(6-13)}$. De hecho, el primer caso de infección en el sistema nervioso central (SNC) por esta bacteria fue en 1997, un niño con ependimoma ${ }^{(7)}$. El segundo reporte de infección del SNC fue en un paciente en Taiwán al cual se le colocó un catéter de ventriculostomía ${ }^{(8)}$. El caso más recientemente publicado fue un neonato con distrés que desarrolló bacteriemia por $E$. brevis ${ }^{(6)}$. Por lo tanto, nuestro caso es la cuarta infección del SNC en las especies humanas. En nuestra experiencia el paciente fue diagnosticado con ventriculitis. Esta afección puede ser consecuencia de una infección del SNC o puede ser adquirida en un $6-38 \%$ después de un procedimiento neurológico invasivo ${ }^{(14)}$.

Como los informes anteriores, la fuente de infección parece ser nosocomial. Esta premisa se apoya en diversos estudios $^{(7-10)}$. Uno de ellos identificó que 11 de 12 pacientes desarrollaron endoftalmitis posterior a cirugía de catarata por el mismo cirujano. Por lo tanto, se sospechó que un problema con el proceso de esterilización era la causa de la enfermedad ${ }^{(10)}$. Además, Raman et al., reportaron una mujer de 65 años la cual tuvo una herida cortante en la rodilla y fue suturada. Posteriormente desarrolló bacteriemia causada por Empedobacter brevis, por lo que la fuente probable de infección fueron los instrumentos empleados para suturar la herida ${ }^{(9)}$. En nuestro caso, la fuente de infección fue probablemente durante la cesárea. Esta evidencia da como resultado que esta bacteria tiene una relación con el proceso de esterilización de instrumentos en establecimientos de salud; no obstante, se necesitan más investigaciones para confirmar esta afirmación.

El tratamiento varía en todos los previos reportes. Por ejemplo, reportes previos afirman una adecuada respuesta con hidrocloruro de minociclina y piperacilina-tazobactam más ciprofloxacina en infecciones dérmicas ${ }^{(11,13)}$. En nuestro caso, la prueba inicial de laboratorio mostró bacterias gramnegativas; por lo tanto, se utilizó un tratamiento empírico con antibióticos de amplio espectro, como el meropenem y la vancomicina, debido a un riesgo de infección con bacterias nosocomiales de alta resistencia. Duong et al. (7). siguieron una terapia similar; sin embargo, a diferencia de su situación, el caso presentado presentaba una infección bacteriana resistente al meropenem e imipenem, por lo que el tratamiento cambió a piperacilina-tazobactam. A pesar de esta medicación, los síntomas del paciente y las pruebas de laboratorio no mejoraron. Por lo tanto, el tratamiento con antibióticos se desplazó a colistina y meropenem. En relación con la literatura anterior para esta bacteria, el patrón sensible no ha cambiado aparentemente, teniendo en cuenta que tiene una distribución mundial (Tabla 3).

Por otra parte, Arnold-Chiari tipo II es una afección rara, relacionada con hidrocefalia y mielomeningocele. Por ejemplo, Matozinho et al. reportó un caso de un neonato que tenía estas tres condiciones, además desarrolló convulsiones a través de días consecutivos ${ }^{(15)}$. Nuestro paciente tenía las enfermedades anteriores mencionadas, pero las convulsiones eran más intermitentes y prolongadas. A pesar de esto, su condición mejoró y fue dado de alta en su día 90 de vida

En conclusión, se presenta el caso de un recién nacido con ventriculitis por un bacilo denominado Empedobacter brevis. El paciente tenía un diagnóstico prenatal de mielomeningocele e hidrocefalia, por lo que fue remitido a nuestro instituto para tratamiento especializado. Según la literatura, E. brevis tiene una distribución mundial, y los pacientes pueden infectarse dentro de los establecimientos de salud; por lo tanto, este bacilo podría tener una alta resistencia a los antibióticos de amplio espectro. Además, este informe da lugar a una nueva etiología de la infección del SNC en esta población en específico, y la necesidad de una correcta identificación del patrón resistente a los antibióticos, con el fin de dar un adecuado manejo terapéutico.

Fuentes de financiamiento: Autofinanciado.

Conflictos de interés: Los autores declaran no tener conflictos de interés en la publicación de este artículo.

Contribución de los autores: CRC, GDCK, MEES, BVV participaron en la concepción y diseño del artículo; recolección de resultados; redacción del artículo; revisión crítica del artículo y aprobación de la versión final. CRC facilitó el aporte de las imágenes y obtención de datos clínicos del paciente.

\section{REFERENCIAS BIBLIOGRÁFICAS}

1. Bernardet J-F, Segers P, Vancanneyt M, Berthe F, Kersters K, Vandamme P. Cutting a Gordian Knot: Emended Classification and Description of the Genus Flavobacterium, Emended Description of the Family Flavobacteriaceae, and Proposal of Flavobacterium hydatis nom. nov. (Basonym, Cyto- phaga aquatilis Strohl and Tait 1978). Int J Syst Evol Microbiol. 1996;46(1):128-48.

2. Jooste PJ, Hugo CJ. The taxonomy, ecology and cultivation of bacterial genera belonging to the family Flavobacteriaceae. Int J Food Microbiol. 1999;53(2-3):81-94.
3. Bernardet JF, Nakagawa Y, Holmes B. Proposed minimal standards for describing new taxa of the family Flavobacteriaceae and emended description of the family. Int J Syst Evol Microbiol. 2002;52(Pt 3):1049-70.

4. Bellais S, Girlich D, Karim A, Nordmann P. EBR-1, a novel Ambler subclass 
B1 beta-lactamase from Empedobacter brevis. Antimicrob Agents Chemother. 2002;46(10):3223-7.

5. Moulin V, Freney J, Hansen W, Philippon A. Comportement phénotypique des Flavobacterium vis-à-vis de 39 antibiotiques. Med Mal Infect. 1992;22(11):902-7.

6. Sharma D, Patel A, Soni P, Sharma P, Gupta B. Empedobacter brevis Meningitis in a Neonate: A Very Rare Case of Neonatal Meningitis and Literature Review. Case Rep Pediatr. 2016;2016:7609602.

7. Duong M, Mourier K, Peyrard N, Magnin V, Couillaud G, Chavanet P. Méningite à Chryseobacterium (Flavobacterium) après intervention neuro-chirurgicale. A propos de deux cas. Med Mal Infect. 1997;27(89):802-3.

8. Chi H, Chang KY, Chang HC, Chiu NC, Huang FY. Infections associated with indwelling ventriculostomy cathe- ters in a teaching hospital. Int J Infect Dis. 2010;14(3):e216-9.

9. Raman S, Shaaban H, Sensakovic JW, Perez G. An Interesting Case of Empedobacter Brevis Bacteremia After Right Knee Cellulitis. J Glob Infect Dis. 2012;4(2):136-7.

10. Janknecht P, Schneider CM, Ness T. Outbreak of Empedobacter brevis endophthalmitis after cataract extraction. Graefes Arch Clin Exp Ophthalmol. 2002;240(4):291-5.

11. Nishio E. [A case of Anaphylactoid purpura suggested to Empedobacter (flavobacterium) brevis infection concerned]. Arerugi. 2010;59(5):558-61.

12. HaburjakJJ,Schubert TA. Flavobacterium breve meningitis in a dog. J Am Anim Hosp Assoc. 1997;33(6):509-12.

13. Bokhari S, Abbas N, Singh M, Cindrich RB, Zeana C. Empedobacter brevis
Bacteremia in a Patient Infected with HIV: Case Report and Review of Literature. Case Rep Infect Dis. 2015;2015:1-3

14. Nieto del Rincón N, de Alba Romero C, Egea Nadal P, et al. Ventriculitis: experiencia en un servicio de neonatología. An Esp Pediatr. 2000;52(3):245-50.

15. Matozinho HHS, Rosa JM, Ferreira JA, et al. Case report of a newborn with ArnoldChiari malformation type Iiassociated with hydrocephalus and myelomeningocele. J Neurol Sci. 2015;357, Supplement 1:e204.

Correspondencia: Bryan Valcarcel Valdivia Dirección: Psje. Huamanga 216, Balconcillo, La Victoria. Lima, Perú.

Correo electrónico:bryan.valcarcel@gmail.com 